\title{
Dinâmica da Transmissão da Dengue com Dados Entomológicos Temperatura-dependentes ${ }^{1}$
}

H.M. YANG ${ }^{2}$, Departamento de Matemática Aplicada, Instituto de Matemática, Estatística e Computação Científica, UNICAMP e Lab-Epifisma, Cx.P. 6065, 13081-970 Campinas, SP, Brasil

M.L.G. MACORIS, K.C. GALVANI, M.T.M. ANDRIGHETTI, Superintendência de Controle de Endemias, Avenida Santo Antonio, 1627, Bairro Somenzari, 17506-040 Marília, SP, Brasil.

Resumo. Dengue é uma doença viral transmitida por artrópodos, os mosquitos Aedes aegypti. Dengue apresenta ciclos epidemiológicos característicos com periodicidade em torno de 2 anos, mas apresenta flutuações anuais devido às variações sazonais. Como a temperatura afeta a incidência? Essa questão é respondida aplicando teoria da dinâmica populacional que fornece o valor do número de reprodutibilidade basal. Estuda-se esse valor em função de temperatura.

\section{Introdução}

O vírus da dengue, da família flavivirus, é transmitido por artrópodos de gênero Aedes e ocorre em áreas densamente povoadas. Por causa de epidemias com conseqüências sérias tornou-se um dos maiores problemas de saúde pública em regiões tropicais e sub-tropicais onde mosquitos Aedes aegypti estão presentes [4]. A própria distribuição geográfica do vetor transmissor da dengue mostra a forte dependência com temperatura e humidade (chuva). Nos períodos quentes e chuvosos ocorrem um aumento da densidade de mosquitos, resultando, como conseqüência, maior incidência de dengue.

O conhecimento das causas que influenciam na variação no número da população de mosquitos [5] pode auxiliar na escolha da forma de controle mais apropriada para diminuir a densidade de mosquitos. As formas de controle comumente aplicadas são mecânica (remoção de criadouros) e química (larvicida e inseticida). Mosquito Aedes aegypti apresenta no seu ciclo de vida dois ambientes distintos: aquática, que compreende fases ovo, larva e pupa, e alada (fase adulta). Em cada uma das fases ovo, larva, pupa e adulta ocorrem mortalidades específicas. Há também tempos de transição distintos da fase ovo para fase larva, de larva para pupa, e de pupa para mosquito adulto. Finalmente, é preciso determinar a capacidade de oviposição das

\footnotetext{
${ }^{1}$ Apoio financeiro FAPESP (Projeto Temático)

${ }^{2}$ hyunyang@ime.unicamp.br, bolsista CNPq.
} 
fêmeas. Todos esses parâmetros entomológicos dependem de temperatura [1] [2] [6]. $\mathrm{Na}$ teoria de dinâmica populacional, o número de indivíduos de uma comunidade é determinado ao se conhecer os parâmetros que regem natalidade, mortalidade, migração e transição entre diferentes fases do ciclo de vida.

Nesse trabalho, utiliza-se os dados entomológicos relacionados com o ciclo de vida de $A$. aegypti para determinar o número de mosquitos. Com respeito à transmissão de dengue, determina-se a razão de reprodutibilidade basal e se estuda a influência da temperatura. Na secção 2 apresenta-se o modelo, que é analisado na secção 3. Conclusão é dada na secção 4.

\section{Modelo Matemático}

Desenvolve-se um modelo matemático para descrever a transmissão de dengue. Primeiro, aborda-se o ciclo de vida de $A$. aegypti. Não se considera a fase de ovo. $\mathrm{O}$ número de larvas em cada instante de tempo é representado por $L(t)$. A quantidade de larvas aumenta com a eclosão de ovos, que as fêmeas adultas põem em criadouros disponíveis para recebê-los, e diminui com a transformação destas em pupas e com a morte. A taxa intrínseca de oviposição e as taxas per-capitas de transformação e de morte são designadas, respectivamente, por $\phi, \delta$ e $\mu_{l}$. A taxa efetiva de produção total de larvas é dada por $\varphi(W)(1-L / C)$, onde $\varphi(W)$, que depende da taxa intrínseca de oviposição $\phi$ e do número de fêmeas $W$, é a capacidade de produção de larvas, a partir de ovos viáveis, por todas as fêmeas e $(1-L / C)$ é a disponibilidade de criadouros para receber as larvas que eclodem de ovos, com $C$ sendo a capacidade total de criadouros. O número de pupas é representado por $P(t)$. A quantidade de pupas aumenta com a transformação de larvas, e diminui com a eclosão (ou emergência) destes em mosquitos adultos e com a morte. As taxas per-capitas de eclosão para a fase adulta e de mortalidade são designadas, respectivamente, por $\sigma$ e $\mu_{p}$. O número total de mosquitos adultos fêmeas $W(t)$ aumenta com a eclosão (ou emergência) de pupas, e diminui com a morte. A taxa per-capita de mortalidade de fêmeas adultas é designada por $\mu_{w}$.

Descreve-se agora o ciclo epidêmico suscintamente. As fêmeas de mosquitos são infectadas durante o repasto sangüíneo nos indivíduos infectantes, e tornam-se infectantes após um período de incubação (extrínseca) entre 7 e 10 dias. Depois desse tempo de incubação, elas transmitem a infecção por toda a sua vida, pois resposta imunológica entre mosquitos é limitada. Esses mosquitos infectantes transmitem o vírus, novamente durante o repasto sangüíneo, para indivíduos suscetíveis que, depois de um período de incubação (intrínseca) entre 4 a 6 dias, manifestam a doença. As formas de doença podem ser dengue clássica ou dengue hemorrágica, que perduram entre 3 e 7 dias (período infeccioso). Depois desse período a resposta imunológica induzida perdura por toda a vida sem enfraquecer. O modelo não vai supor que os períodos de incubação extrínseca e intrínseca e o período infeccioso dependam de temperatura, além de considerar infecção por um único sorotipo.

$\mathrm{O}$ vírus da dengue circula na comunidade quando há interação entre populações humana e de mosquito. Considerando a história natural da doença, a população humana é sub-dividida em quatro compartimentos não-interceptantes: suscetíveis, 
expostos, infectantes e recuperados. Assume-se que a população humana é constante, onde o número total de indivíduos é $N$. Assim, as frações de indivíduos suscetíveis, expostos, infectantes e recuperados em cada instante de tempo $t$ são dadas por $s(t), e(t), i(t)$ e $r(t)$, respectivamente. Os indivíduos suscetíveis são infectados pelos mosquitos infectantes e passam para a classe dos expostos proporcionalmente a uma taxa de freqüência com que mosquitos picam humanos (genericamente referido como taxa de contato per-capita $\beta_{h}^{\prime}$ ). Os indivíduos expostos são retirados deste compartimento para infectantes a uma taxa $\alpha$, onde $\alpha^{-1}$ é o período de incubação intrínseca. Por sua vez, esses indivíduos infectantes são retirados deste compartimente para recuperados a uma taxa $\eta$, onde $\eta^{-1}$ é o período de infecção (ou recuperação) do indivíduo humano. Não se consideram a perda de imunidade e a presença de anticorpos maternos. Todos os indivíduos estão sob a influência de uma mesma taxa de mortalidade dada por $\mu$ e não há mortalidade adicional pela doença, uma vez que se está considerando infecção por um único sorotipo. Por se tratar de infecção por um único sorotipo, a imunidade é perene.

Quanto à população de mosquitos adultos, ela é sub-dividida em mosquitos suscetíveis $\left(W_{1}\right)$, infectados porém não infectantes $\left(W_{2}\right)$ e infectantes $\left(W_{3}\right)$. Mosquitos suscetíveis são infectados quando picam indivíduos infectantes, proporcionalmente a uma taxa de contato total $\beta_{w}$, que é o produto entre número total de humanos $N$ e taxa per-capita $\beta_{w}^{\prime}$. Os mosquitos infectados e não infectantes são removidos para a classe de infectantes a uma taxa $\gamma$, onde $\gamma^{-1}$ é o período de incubação extrínseca do vírus no mosquito. O número total de mosquitos é $W=W_{1}+W_{2}+W_{3}$.

Com as considerações acima, a dinâmica de transmissão da dengue na população de mosquitos é descrita por

$$
\left\{\begin{array}{l}
\frac{d}{d t} L(t)=\phi W(t)\left[1-\frac{L(t)}{C}\right] L(t)-\left(\delta+\mu_{l}\right) L(t) \\
\frac{d}{d t} P(t)=\delta L(t)-\left(\sigma+\mu_{p}\right) P(t) \\
\frac{d}{d t} W_{1}(t)=\sigma P(t)-\left[\beta_{w} i(t)+\mu_{w}\right] W_{1}(t) \\
\frac{d}{d t} W_{2}(t)=\beta_{w} i(t) W_{1}(t)-\left(\gamma+\mu_{w}\right) W_{2}(t) \\
\frac{d}{d t} W_{3}(t)=\gamma W_{2}(t)-\mu_{w} W_{3}(t),
\end{array}\right.
$$

e na população humana, é descrita pelo sistema de equações

$$
\left\{\begin{array}{l}
\frac{d}{d t} s(t)=\mu-\left[\varepsilon \beta_{h}^{\prime} W_{3}(t)+\mu\right] s(t) \\
\frac{d}{d t} e(t)=\varepsilon \beta_{h}^{\prime} W_{3}(t) s(t)-(\alpha+\mu) e(t) \\
\frac{d}{d t} i(t)=\alpha e(t)-(\eta+\mu) i(t) \\
\frac{d}{d t} r(t)=\eta i(t)-\mu r(t),
\end{array}\right.
$$

onde $\varepsilon$, com $0<\varepsilon<1$, representa uma redução na busca efetiva por repasto sangüíneo devido ao envelhecimento de mosquitos, e $s(t)+e(t)+i(t)+r(t)=1$. (Primeiro, os mosquitos suscetíveis devem picar indivíduos infectantes, sobreviver o período extrínseco, e, então, como infectantes, picar indivíduo suscetível.) Todos os demais parâmetros e variáveis já foram previamente definidos. Ressalta-se que a duração de uma geração na população de mosquitos é de algumas semanas, sendo no máximo seis meses. Por isso, permitiu-se que a população total de mosquitos adultos $(W)$ variasse no tempo. Comparativamente, a duração de uma geração entre 
os indivíduos humanos é de dezenas de anos, o que permite considerar a população humana $(N)$ constante no tempo.

A força de infecção $\lambda_{h}(t)$, nome dada para incidência per-capita, de dengue entre humanos depende da taxa de contato entre humanos e mosquitos e quantidade de mosquitos infectantes, $\lambda_{h}(t)=\varepsilon \beta_{h}^{\prime} W_{3}(t)$, e mede o risco, por unidade de tempo, de uma pessoa adquirir a doença. Multiplicando a força de infecção pelo número total de suscetíveis tem-se o número de novos casos de infecção entre humanos por unidade de tempo (incidência total), $\lambda_{h}(t) s(t) N$. Analogamente, tem-se a força de infecção de dengue entre mosquitos dada por $\lambda_{w}(t)=\beta_{w} i(t)=\beta_{w}^{\prime} N i(t)$, e a incidência total, $\lambda_{w}(t) W_{1}(t)$. Percebe-se, assim, que o modelo acima assumiu para a incidência produto entre duas sub-populações, $W_{3}(t)$ e $s(t) N$ ou $W_{1}(t)$ e $i(t) N$. Em algumas situações o que importa não é o número absoluto, mas o valor relativo (densidade) das sub-populações na propagação da infecção. Nesse tipo de formulação, uma vez que se está abordando modelos com parâmetros constantes, as taxas de contato utilizados devem ser totais (ou populacionais), e constantes no tempo. Note que para incidência de dengue entre mosquitos pode-se utilizar indistintamente taxa de contato per-capita ou total (da relação $\beta_{w}=N \beta_{w}^{\prime}$, pois a população humana é constante), o que não é verdade para a incidência de dengue entre humanos (população de mosquitos varia com o tempo).

\section{Análise do Modelo}

O modelo proposto é similar ao apresentado em [7]. O modelo aqui apresentado difere quanto à ausência de compartimento de ovos de mosquitos, assim como na ausência de fator de envelhecimento entre mosquitos. A exclusão de ovos deve-se ao fato dos valores de parâmetros entomológicos obtidos em laboratório não contemplar a fase de ovo [3], e o objetivo é elaborar um modelo fundamentado em valores dos parâmetros conhecidos (ou estimados).

A dinâmica de transmissão de dengue envolvendo duas populações, humana e de mosquito, dada pelas equações (2.1) e (2.2), é estudada no estado estacionário. Primeiro apresenta-se os pontos de equilíbrio, e, derivando da análise de estabilidade desses pontos, obtém-se o número de reprodutibilidade basal. Desse parâmetro epidemiológico, estuda-se os valores limiares dependentes de temperatura.

\subsection{Análise dos pontos de equilíbrio}

São 3 possíveis situações de equilíbrio.

Comunidade livre da infestação de mosquito. Nessa situação, para população de mosquitos, temos os valores em equilíbrio dados por

$$
\left\{\begin{array}{l}
L=P=0 \\
W_{1}=W_{2}=W_{2}=0,
\end{array}\right.
$$

e para população humana,

$$
\left\{\begin{array}{l}
s=1 \\
e=i=r=0 .
\end{array}\right.
$$


Esse ponto de equilíbrio $S^{0}$ é dito comunidade sem mosquito.

Comunidade livre de transmissão de dengue. Nessa situação, para população de mosquitos, temos os valores em equilíbrio dados por

$$
\left\{\begin{aligned}
L & =C\left(1-\frac{1}{Q_{0}}\right) \\
P & =\frac{\delta}{\sigma+\mu_{p}} C\left(1-\frac{1}{Q_{0}}\right) \\
W_{1} & =\frac{\sigma \delta}{\mu_{w}\left(\sigma+\mu_{p}\right)} C\left(1-\frac{1}{Q_{0}}\right) \\
W_{2} & =0=W_{3},
\end{aligned}\right.
$$

onde o número de descendentes basal $Q_{0}$ é definido por

$$
Q_{0}=\frac{\delta}{\delta+\mu_{l}} \times \frac{\sigma}{\sigma+\mu_{p}} \times \frac{\phi}{\mu_{w}},
$$

e para população humana,

$$
\left\{\begin{array}{l}
s=1 \\
e=i=r=0 .
\end{array}\right.
$$

Esse ponto de equilíbrio $S^{f}$ é dito comunidade livre da doença, e só é viável biologicamente se $Q_{0}>1$.

Dengue prevalente na comunidade. Nessa situação, para população de mosquitos, temos os valores em equilíbrio, que estão definidos para $i>0$, dados por

$$
\left\{\begin{aligned}
L & =C\left(1-\frac{1}{Q_{0}}\right) \\
P & =\frac{\left(\gamma+\mu_{w}\right) \mu_{w}^{2}(\alpha+\mu)(\eta+\mu)\left(1+\frac{\beta_{w}}{\mu_{w}} i\right)}{\varepsilon \beta_{h}^{\prime} \beta_{w} \alpha \gamma \sigma\left[1-\frac{(\alpha+\mu)(\eta+\mu)}{\alpha \mu}\right]} \\
W_{1} & =\frac{\left(\gamma+\mu_{w}\right) \mu_{w}(\alpha+\mu)(\eta+\mu)\left(1+\frac{\beta_{w}}{\mu_{w}} i\right)}{\varepsilon \beta_{h}^{\prime} \beta_{w} \alpha \gamma\left[1-\frac{(\alpha+\mu)(\eta+\mu)}{\alpha \mu} i\right]}-\frac{\left(\gamma+\mu_{w}\right)(\alpha+\mu)(\eta+\mu) i}{\varepsilon \beta_{h}^{\prime} \alpha \gamma\left[1-\frac{(\alpha+\mu)(\eta+\mu)}{\alpha \mu} i\right]} \\
W_{2} & =\frac{\mu_{w}(\alpha+\mu)(\eta+\mu) i}{\varepsilon \beta_{h}^{\prime} \alpha \gamma\left[1-\frac{(\alpha+\mu)(\eta+\mu)}{\alpha+\mu}\right]} \\
W_{3} & =\frac{(\alpha+\mu)(\eta+\mu) i}{\varepsilon \beta_{h}^{\prime} \alpha\left[1-\frac{(\alpha+\mu)(\eta+\mu)}{\alpha \mu} i\right]}
\end{aligned}\right.
$$

e para população humana,

$$
\left\{\begin{aligned}
s & =1-\frac{(\alpha+\mu)(\eta+\mu)}{\alpha \mu} i \\
e & =\frac{\eta+\mu}{\alpha} i \\
i & =\frac{R_{0}-1}{\frac{\beta w}{\mu}+\frac{(\alpha+\mu)(\eta+\mu)}{\alpha \mu} R_{0}} \\
r & =\frac{\eta}{\mu} i
\end{aligned}\right.
$$

onde o número de reprodutibilidade basal $R_{0}$ é definido por

$$
R_{0}=\bar{R}_{0} \times \frac{\delta C\left(1-\frac{1}{Q_{0}}\right)}{\mu_{w}} \times \frac{\sigma}{\sigma+\mu_{p}},
$$

com $\bar{R}_{0}$ sendo dado por

$$
\bar{R}_{0}=\frac{\gamma}{\gamma+\mu_{w}} \times \frac{\varepsilon \beta_{h}^{\prime}}{\mu_{w}} \times \frac{\alpha}{\alpha+\mu} \times \frac{\beta_{w}}{\eta+\mu} .
$$


Esse ponto de equilíbrio $S^{d}$ é dito dengue prevalente na comunidade, e só é viável biologicamente se $Q_{0}>1$ e $R_{0}>1$.

A análise de estabilidade segue o mesmo padrão do modelo apresentado em [7]. O ponto de equilíbrio correspondente a comunidade sem mosquito é localmente e assintoticamente estável para $Q_{0}<1$. O ponto de equilíbrio correspondente a comunidade livre da doença é localmente e assintoticamente estável para $Q_{0}>1$ e $R_{0}<1$. A doença prevalente na comunidade é localmente e assintoticamente estável para $Q_{0}>1$ e $R_{0}>1$.

As bifurcações no espaço de parâmetros $\left(Q_{0}, R_{0}\right)$ ocorrem com respeito às retas $Q_{0}=1$ e $R_{0}=1$, que dividem o primeiro quadrante em 4 regiões retangulares. Na região I $\left(Q_{0}<1\right.$ e $\left.R_{0}<1\right)$ e na região II $\left(Q_{0}<1\right.$ e $\left.R_{0}>1\right)$ o único ponto de equilíbrio é o $S^{0}$, que é estável. Quando passa de região I para II pela fronteira (reta $\left.R_{0}=1\right)$, não ocorre bifurcação. Na região III $\left(Q_{0}>1\right.$ e $\left.R_{0}<1\right)$ existem dois pontos de equilíbrio $S^{0}$ e $S^{f}$, este é estável e o outro, instável. Quando passa de região I para III pela fronteira (reta $Q_{0}=1$ ), ocorre bifurcação: surge $S^{f}$ estável e $S^{0}$ torna-se instável. Na região IV $\left(Q_{0}>1\right.$ e $\left.R_{0}>1\right)$ existem três pontos de equilíbrio $S^{0}, S^{f}$ e $S^{d}$, este último é estável e os outros, instáveis. Quando passa de região II para IV pela fronteira (reta $Q_{0}=1$ ), ocorre bifurcação: surge $S^{d}$ estável e $S^{0}$ torna-se instável; porém, quando passa de região III para IV pela fronteira (reta $R_{0}=1$ ), ocorre outra bifurcação: surge $S^{d}$ estável e $S^{f}$ torna-se instável. No ponto de acumulação $\left(Q_{0}=1, R_{0}=1\right)$ podem ocorrer todas as bifurcações. A região II é a mais perigosa, pois pode passar de situação sem mosquitos para dengue endêmico.

Interpreta-se os valores de bifurcação $Q_{0}=1$ e $R_{0}=1$. Entende-se $Q_{0}$, dado pela equação (3.1), como sendo o número médio de mosquitos fêmeas descendentes produzidas por uma única fêmea: um ovo viável eclode, sobrevive fase de larva e transforma-se em pupa com probabilidade $\delta /\left(\delta+\mu_{l}\right)$, sobrevive a fase de pupa e emerge como adulto com probabilidade $\sigma /\left(\sigma+\mu_{p}\right)$ e durante a sua sobrevivência $\left(1 / \mu_{w}\right)$ gera em média $\phi / \mu_{w}$ ovos viáveis. Por isso o limiar de infestação de mosquitos ocorre quando uma fêmea gera em média uma outra fêmea $\left(Q_{0}=1\right)$.

Para entender $R_{0}$, separa-se em duas etapas. Primeiro, entende-se $\bar{R}_{0}$, dado pela equação (3.3), como sendo o número médio de novos mosquitos infectantes produzidos por um único mosquito infectante: um mosquito suscetível ao se infectar por picar um indivíduo infectante, sobrevive o período de latência e torna-se infectante com probabilidade $\gamma /\left(\gamma+\mu_{w}\right)$, pica e infecta, durante a sua sobrevivência $\left(1 / \mu_{w}\right)$, em média $\varepsilon \beta_{h}^{\prime} / \mu_{w}$ indivíduos suscetíveis; cada indivíduo infectado sobrevive o período latente e torna-se infectante com probabilidade $\alpha /(\alpha+\mu)$, e durante o período infectante $(1 /(\eta+\mu))$ é picado em média por $\beta_{w} /(\eta+\mu)$ mosquitos suscetíveis que se infectam. Na segunda parte, entende-se os dois últimos termos da equação (3.2) como sendo a quantidade de novos mosquitos (adultos) que podem vir a ser infectados: o número de mosquitos é dado pelo número de larvas $\left(C\left(1-1 / Q_{0}\right)=L\right)$ eclodidas de ovos produzidos durante a sobrevivência de um mosquito adulto $\left(1 / \mu_{w}\right)$, que se tornam pupas $\left(\delta C\left(1-1 / Q_{0}\right) / \mu_{w}\right)$, e sobrevivem a fase pupa emergindo como mosquitos adultos com probabilidade $\sigma /\left(\sigma+\mu_{p}\right)$. Assim, a infecção de dengue é prevalente na comunidade se um mosquito infectante gerar em média outro mosquito infectante $\left(R_{0}=1\right)$. Note que, no caso de incidência definida como produto de densidades de sub-populações, o número de reproduti- 
bilidade basal é o próprio $\bar{R}_{0}$, que depende somente de parâmetros relacionados com a doença. Quando a incidência é estruturada em termos de números de subpopulações, o número de reprodutibilidade basal é $R_{0}$, que depende de po-pulação de mosquitos, além dos parâmetros relacionados com a doença.

\subsection{Valores limiares dependentes de temperatura}

Os valores basais $Q_{0}$ e $R_{0}$ (também, $\bar{R}_{0}$ ) dependem de parâmetros entomológicos e da história natural da infecção de dengue. Mostra-se como os riscos de infestação de mosquitos e de emergência de dengue dependem de temperatura. Antes esses valores são re-escritos em termos de valores limiares.

O número de descendentes basal $Q_{0}$, dado pela equação (3.1), determina o nível de infestação de mosquitos, como também o limiar da bifurcação em $Q_{0}=1$. A condição $Q_{0}>1$ pode ser re-escrita como

$$
\phi>\phi^{t h} \equiv \frac{\left(\delta+\mu_{l}\right)\left(\sigma+\mu_{p}\right) \mu_{w}}{\delta \sigma} .
$$

Logo, $\phi=\phi^{\text {th }}$ (unidade tempo ${ }^{-1}$ ) é o limiar da taxa de oviposição para ocorrer infestação de mosquitos.

O número de reprodutibilidade basal $R_{0}$, dado pela equação (3.2), determina o nível de epidemia de dengue, como também o limiar da bifurcação em $R_{0}=1$. A condição $R_{0}>1$ pode ser re-escrita como

$$
\varepsilon \beta_{h}^{\prime} \beta_{w}>\beta^{t h} \equiv \frac{\left(\sigma+\mu_{p}\right)\left(\gamma+\mu_{w}\right) \mu_{w}^{2}(\alpha+\mu)(\eta+\mu)}{\gamma \alpha \delta \sigma C\left(1-\frac{\phi^{t h}}{\phi}\right)} .
$$

Logo, $\varepsilon \beta_{h}^{\prime} \beta_{w}=\beta^{\text {th }}$ (unidade tempo ${ }^{-2}$ ) é o limiar da transmissão para ocorrer epidemia de dengue. No caso em que a incidência é estruturada em termos de densidades, o número de reprodutibilidade basal $\bar{R}_{0}$, dada pela equação (3.3), determina o nível de epidemia de dengue, como também o limiar da bifurcação em $\bar{R}_{0}=1$. A condição $\bar{R}_{0}>1$ pode ser re-escrita como

$$
\varepsilon \beta_{h}^{\prime} \beta_{w}>\bar{\beta}^{t h} \equiv \frac{\left(\gamma+\mu_{w}\right) \mu_{w}(\alpha+\mu)(\eta+\mu)}{\gamma \alpha} .
$$

Logo, $\varepsilon \beta_{h}^{\prime} \beta_{w}=\bar{\beta}^{\text {th }}$ (unidade tempo ${ }^{-2}$ ) é o limiar da transmissão para ocorrer epidemia de dengue.

Os parâmetros entomológicos, que compõem maior número de parâmetros do modelo, são obtidos de experimentos laboratorias. Em uma câmara de germinação, a uma temperatura fixa, é acompanhado um grupo de mosquitos (ou larvas), anotandose a cada 24 horas o número de transições para outra fase (caso da fase aquática) e de morte. Esses dados são ajustados para extrair parâmetros de transição e de mortalidade das diversas fases do ciclo de vida de mosquito. As taxas dependentes de temperatura $T\left(\mathrm{em}^{0} \mathrm{C}\right)$ são representadas pelo polinômio de grau $m$,

$$
\Theta_{m}(T)=\sum_{i=0}^{m} b_{i} T^{i}
$$




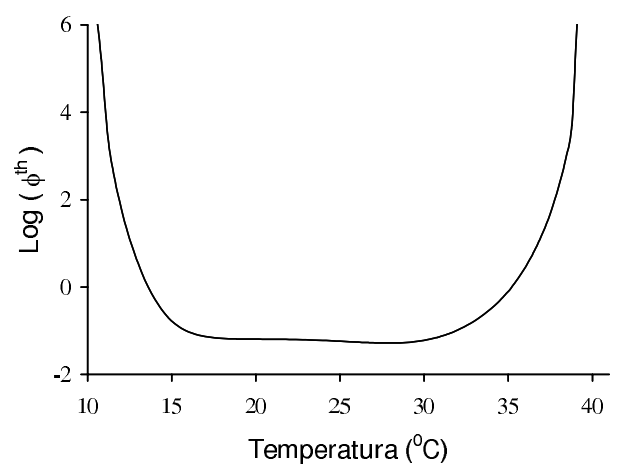

Figura 1: A curva de $\phi^{t h}(T)$ dividindo regiões com $\left(\phi>\phi^{t h}\right)$ e sem $\left(\phi<\phi^{t h}\right)$ mosquitos.

onde $b_{i}$ (unidade $\operatorname{dias}^{-1} \times\left[{ }^{0} C\right]^{-i}$ ), com $i=0,1,2, \cdots, m$, são os coeficientes ajustados em [3]. Os coeficientes para taxa de oviposição $\phi$ são $b_{0}=-1,213 \times 10^{1}$, $b_{1}=2,519, b_{2}=-2,020 \times 10^{-1}, b_{3}=7,935 \times 10^{-3}$ e $b_{4}=-1,064 \times 10^{-4}$. Os coeficientes para taxas de transição $\delta$ (larva para pupa) e $\sigma$ (pupa para adulto) são, respectivamente, $b_{0}=3,075 \times 10^{-1}, b_{1}=-1,425 \times 10^{-1}, b_{2}=2,835 \times 10^{-2}$, $b_{3}=-3,047 \times 10^{-3}, b_{4}=1,842 \times 10^{-4}, b_{5}=-6,078 \times 10^{-6}, b_{6}=1,024 \times 10^{-7}$, $b_{7}=-7,168 \times 10^{-10}$ e $b_{8}=6,052 \times 10^{-13} ;$ e $b_{0}=-3,164, b_{1}=1,146, b_{2}=$ $-1,587 \times 10^{-1}, b_{3}=1,037 \times 10^{-2}, b_{4}=-3,086 \times 10^{-4}, b_{5}=2,282 \times 10^{-6}$, $b_{6}=8,201 \times 10^{-8}, b_{7}=-1,859 \times 10^{-9}$ e $b_{8}=1,102 \times 10^{-11}$. Os coeficientes das taxas de mortalidades $\mu_{l}, \mu_{p}$ e $\mu_{w}$ são, respectivamente, $b_{0}=2,315, b_{1}=$ $-4,191 \times 10^{-1}, b_{2}=2,735 \times 10^{-2}, b_{3}=-7,538 \times 10^{-4}$ e $b_{4}=7,503 \times 10^{-6}$; $b_{0}=0,426, b_{1}=-0,324 \times 10^{-1}, b_{2}=0,706 \times 10^{-3}$ e $b_{3}=0,440 \times 10^{-6} ; \mathrm{e}$ $b_{0}=8,692 \times 10^{-1}, b_{1}=-1,590 \times 10^{-1}, b_{2}=1,116 \times 10^{-2}, b_{3}=-3,408 \times 10^{-4} \mathrm{e}$ $b_{4}=3,809 \times 10^{-6}$.

A Figura 1 mostra o limiar de infestação $\phi^{\text {th }}$ em função de temperatura. Assumese que a capacidade total de criadouros não depende de temperatura, $\operatorname{com} C=1$. A curva divide duas regiões quanto a infestação de mosquitos: abaixo da curva estão as regiões livres de infestação de mosquitos; acima, regiões propícias para invasão e colonização por mosquitos.

A Figura 2 mostra os limiares epidêmicos $\beta^{\text {th }}$ e $\bar{\beta}^{\text {th }}$ em função de temperatura. Assume-se que os parâmetros da infecção não dependem de temperatura. Esses valores são: $\gamma=0,125 ; \alpha=0,2$ e $\eta=0,25\left(\mathrm{em} \mathrm{dias}^{-1}\right)$. A taxa constante de mortalidade humana é $\mu=4,3 \times 10^{-5}$ dias $^{-1}$. Apenas parâmetros entomológicos dependem de temperatura. A curva de $\beta^{\text {th }}$ divide duas regiões quanto a prevalência de dengue: abaixo da curva estão as regiões livre de doença; acima, regiões onde a doença estabelece-se endemicamente. O valor atribuído para $C$ desloca a curva de $\beta^{\text {th }}$ em $\log (C)$ unidades (para cima ou para baixo, dependendo do valor de $C$ ). Entretanto, para $\bar{\beta}^{\text {th }}$, abaixo da curva estão as regiões livre de doença; porém, acima da curva a doença pode se estabelecer endemicamente somente se $Q_{0}>1$.

A curva de $\beta^{\text {th }}$ é dependente de temperatura, pois depende de todos os parâmetros 


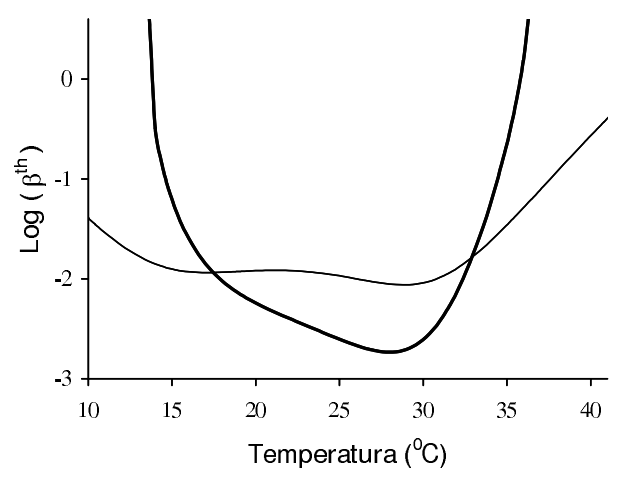

Figura 2: A curva (grossa) de $\beta^{\text {th }}(T)$ e regiões com $\left(\varepsilon \beta_{h}^{\prime} \beta_{w}>\beta^{\text {th }}\right)$ e $\operatorname{sem}\left(\varepsilon \beta_{h}^{\prime} \beta_{w}<\beta^{\text {th }}\right)$ dengue. Porém a curva (fina) de $\bar{\beta}^{\text {th }}(T)$ necessita da condição adicional $\left(\phi>\phi^{\text {th }}\right)$.

entomológicos, e, também, de capacidade total de criadouros. A curva mostrada na Figura 2 utilizou $C=1$; porém, se aumentar para 10, a curva desloca para baixo em 1 unidade, diminuindo a região de erradicação da doença. No entanto, a curva de $\bar{\beta}^{\text {th }}$ depende de temperatura apenas pela taxa de mortalidade de mosquitos adultos, por isso mostra relativamente pequena variação com temperatura. Porém, para que dengue mantenha sua transmissão em nível endêmico, é preciso a presença de vetor interagindo na comunidade. Assim, modelo com incidência estruturada em densidades requer, para dengue prevalente, as condições $\varepsilon \beta_{h}^{\prime} \beta_{w}>\bar{\beta}^{t h}$ e $\phi>\phi^{t h}$, que correspondendem às regiões acima das curvas nas Figuras 1 e 2.

\section{Conclusão}

Um modelo para transmissão de dengue foi desenvolvido considerando incidência estruturada em termos de sub-populações infectantes. O modelo forneceu os números de descendentes basal $Q_{0}$ e de reprodutibilidade basal $R_{0}$, que foram estudados com parâmetros entomológicos dependentes de temperatura.

O risco de colonização e infestação por mosquitos é muito elevado para temperaturas entre 16 e $23{ }^{\circ} \mathrm{C}$, intervalo em que o valor limiar de oviposição $\phi^{\text {th }}$ é menor (Figura 1). Porém, temperaturas com maior risco de deflagração de dengue situa-se entre 25 e $30^{0} C$, intervalo em que o valor limiar de transmissão $\beta^{\text {th }}$ tem menores valores (Figura 2). Discute-se suscintamente sobre a erradicação de dengue. Se o valor limiar de transmissão de dengue for dado por $\bar{\beta}^{\text {th }}$, então a única forma de controle será a aplicação de inseticida, que altera a taxa de mortalidade natural do mosquito $\mu_{w}$ para $\mu_{w}+\mu_{w}^{\prime}$, onde $\mu_{w}^{\prime}$ é mortalidade adicional pelo inseticida. Entretanto, se utilizar incidência proporcional a números de sub-populações, então o valor limiar será dado por $\beta^{t h}$, e aplicações de inseticida $\left(\mu_{w}\right.$ para $\left.\mu_{w}+\mu_{w}^{\prime}\right)$ e larvicida ( $\mu_{l}$ para $\mu_{l}+\mu_{w}^{\prime}$, uma vez que pupas são resistentes) e, também, remoção de criadouros $(C$ para $(1-f) C$, onde $f$ é fração de criadouros removidos) contribuirão 
para a erradicação da doença. No caso de modelo estruturado com densidades, as duas últimas formas de controle apenas diminuiriam o nível de infestação de mosquitos. Porém, se diminuir o valor de limiar de oviposição para abaixo de limiar $\phi^{t h}$, então dengue é erradicada pela erradicação da população de mosquitos.

\begin{abstract}
Dengue is a vector-borne viral disease transmitted by mosquitoes Aedes aegypti. Dengue outbreaks present epidemic cycles of around 2 years, but annually shows the incidence ruled according to seasonal variations. How does the temperature affect the incidence? We address this question applying dynamic population theory from which we yield the basic reproduction number. This number, which depends on the entomological parameters, is analyzed as a function of temperature.
\end{abstract}

\title{
Referências
}

[1] D.A. Focks, D.G. Haile, E. Daniels, G.A. Mount, Dynamic Life Table Model for Aedes aegypti (Diptera:Culicidae): Analysis of the Literature and Model Development, Journal of Medical Entomology, 30 (1993), 1003-1017.

[2] D.A. Focks, D.G. Haile, E. Daniels, G.A. Mount, Dynamic Life Table Model for Aedes aegypti (Diptera:Culicidae): Simulation Results and Validation, Journal of Medical Entomology, 30 (1990), 1018-1028.

[3] M.L.G. Macoris, K.C. Galvani, M.T.M. Andrighetti, D.M.W. Wanderlei, H.M. Yang, Estimating The Entomological Parameters of Mosquito Aedes aegypti from Temperature-Controlled Experimental Data, Ecol. Mod, (2007), submetido.

[4] T.P. Monath (Editor), "Arboviruses: Epidemiology and Ecology", CRC Press, Boca Raton, Florida, 1988.

[5] M.J. Nelson, "Aedes aegypti: Biologia y Ecologia", Organización Panamericana de la Salud, Washington, D.C., 1986.

[6] L.M. Rueda, K.J. Patel, R.C. Axtale, R.E. Stimer, Temperature Development and Survival Rates of Culex quinquefasciatus and Aedes aegypti (Diptera: $\mathrm{Cu}-$ licidae), J. Med. Entomol., 27, No. 5 (1990), 892-898.

[7] H.M. Yang, Epidemiologia da transmissão da dengue, em "Seleta do XXV CNMAC" (E.X.L. de Andrade, J.M. Balthazar, S.M. Gomes, G.N. Siva e A. Sri Ranga, eds.), TEMA - Tendências em Matemática Aplicada e Computacional, Vol. 4, No. 3, pp. 387-396, SBMAC, 2003. 